\title{
The Rise and Fall of the Hydrophobic Effect in Protein Folding and Protein-Protein Association and Molecular Recognition
}

\author{
Arieh Ben-Naim \\ Department of Physical Chemistry, The Hebrew University of Jerusalem, \\ Edmond J. Safra Campus, Givat Ram, Jerusalem, Israel \\ E-mail:arieh@fh.huji.ac.il \\ Received September 14, 2011; October 10, 2011; October 17, 2011
}

\begin{abstract}
In the beginning everything was explained in Biochemistry in terms of hydrogen-bonds (HB). Then, the devastating blow, known as the HB-inventory argument came; hydrogen bonding with water molecules compete with intramolecular hydrogen-bonds. As a result, the HBs paradigm fell from grace. The void that was created immediately filled by Kauzmann's idea of hydrophobic $(H \phi O)$ effect which reigned supreme in biochemical literature for over 50 years (1960-2010). Cracks in the HB-inventory argument on one hand, and doubts about the adequacy of Kauzmann's model for the $H \phi O$ effect, have led to a comeback of the HBs, along with a host of new hydrophilic $(H \phi I)$ effects. The $H \phi O$ effects lost much of its power-which it never really had-in explaining protein folding and protein-protein association. Instead, the more powerful and richer repertoire of $H \phi I$ effects took over the reins. The $H \phi I$ interactions also offered simple and straightforward answers to the problems of protein folding, and protein-protein association.
\end{abstract}

Keywords: Protein Folding, Protein Association, Molecular Recognition, Hydrophobic, Hydrophilic Interactions

\section{Introduction}

This article tells the story of the rise and fall of the hydrophobic $(H \phi O)$ effect in protein folding and protein-protein association. Parallel to this story is the story of the fall and rise of the (hydrophilic) $H \phi I$ effects. The two stories are intertwined; the fall of one led to the rise of the second. The fall of the second led to the comeback of the first. These two stories have also an important moral regarding the mechanism of evolution, survival and extinction of ideas in the biochemical literature, and perhaps in science, in general. The moral is not discussed in this article.

A convenient point to begin with the story is Pauling's book "The Nature of the Chemical Bond" [1,2]. In the first two editions of the book Pauling discussed the HB, but no mention of proteins or nucleic acids. In the second edition, the chapter on HBs (Chapter IX) ends with some estimates of the HB energies and HB distances. The third editions contain two new sections on HBs in proteins and
HBs in nucleic acids.

Following the works of Anson and Mirsky [3] and Mirsky and Pauling [4] on the denaturation of proteins, and latter works of Pauling and Corey [5-8], the role of HBs in stabilizing the native form of proteins became the dogma [9]. The HBs, with bond energies of the order of $24 \mathrm{~kJ} / \mathrm{mol}$, which provided explanation for many anomalous properties of water, also took over the main "cohesive forces needed for the organization of native proteins" [9].

The first cracks in the HB-dogma came from the realization that though the HB energy is the order of 24 $\mathrm{kJ} / \mathrm{mol}$, its formation in aqueous solution must have a far smaller effect on the "driving force" for the process of protein folding. The argument apparently started with the work of Schellman [10,11] summarized by Kauzmann [12] and eventually was encapsulated in Fersht's HBinventory argument [13]. The argument seems simple, straightforward and convincing. Write the stoichiometrical reaction between a donor and an acceptor of a HB in 
the form

$$
\begin{aligned}
& E-C=O \cdots w+w \cdots H N-S \\
& \rightarrow E-C=O \cdots H N-S+w \cdots w
\end{aligned}
$$

where $E$ and $S$ stand for an enzyme and a substract, respectively, but can be any two molecules or parts of the same molecule, and $w$ is a water molecule. Equation (1) suggests that in the process of formation of a direct hydrogen bond between a donor (here amine group) and an acceptor (here a carbonyl group), two HBs are broken on the lhs of the equation and two HBs are formed on the rhs of the equation. Therefore, ignoring the differences in the various HB energies between the various pairs (carbonyl-water, amine-water, carbonyl-amine and water-water), we can conclude by simply counting, that the net effect of the formation of a direct HB is negligibly small. As Kauzmann summarized this argument:

"Hydrogen bonds, taken by themselves, give marginal stability to ordered structures, which may be enhanced or disrupted by interactions of side chains."

We use here the term "driving force" in the sense of $\Delta G<0$ as commonly used in the literature.

If $\mathrm{HBs}$ do not provide the main "driving force" for protein folding, what factors do provide those "driving force?" $[14,15]$. This apparently conceptual vacuum was filled by the $H \phi O$ effect in (1959). The $H \phi O$ effect was known long before Kauzmann applied it to the problem of protein folding [9]. It was applied successfully to explain surface tension of certain aqueous solutions of organic molecules, micelle formation and membranes. All these phenomena involve molecules having two moieties; a hydrophobic part, one "feared" water and tries to avoid it, the second "loved" water and mingled with water comfortably. As Tanford and Reynolds quoted from a personal communication with Kauzmann, the idea of the $H \phi O$ effect was hovering "in the air" for long a long time [9].

In a classical review article "Some Factors in the Interpretation of Protein Denaturation," Kauzmann applied the idea of the $H \phi O$ effect to protein folding [12]. For this purpose he coined the term $\mathrm{H} \phi \mathrm{O}$-bond, and speculated that this "bond" could be the more important factor in the stabilization of the native structure of protein.

Kauzmann's idea was very simple. It was known that the Gibbs energy of transferring a small non-polar solute such as methane or ethane, from water into an organic liquid involves a large negative change in Gibbs energy. This is the same "driving force" that drives the formation of micells and membranes in aqueous solutions. Kauzmann also noticed that there are about one third of amino-acid side-chains which are $H \phi O$, and most of these find themselves in the interior of the folded protein. If one can take the process of transfer, Figure 1(a), to represent the process of transfer of side chain, Figure 1(b), then we can estimate that a protein of about 150 amino acid has about $50 \mathrm{H} \phi \mathrm{O}$ groups, and if each of these contributes between -12 and $-16 \mathrm{~kJ} / \mathrm{mol}$, we get a very large "driving force" for the folding process.

Kauzmann's idea was brilliant. It captured the imagination of many scientists including mine. Add to it the fact that the process of transferring of a non-polar solute from water into an organic liquid is "entropy driven". Add to it that entropy is a mysterious concept [16], not well understood, and you get a "driving force" which is enshrouded with an aura of mystery. Given all these facts, many authors could have claimed that the $H \phi O$ effect is the most important "driving force" in protein folding, without taking any risk of being proved wrong!

How can anyone prove anything on such a complex process as protein folding [15]. Carried out in poorly understood solvent [14], involving a mysterious "entropy driven" concept [10]?

It is not surprising therefore, that the dominance of the $H \phi O$ effect has prevailed for over half a century. The fact that $H \phi O$ groups are in the interior of the protein, and the fact that the transfer of $H \phi O$ molecules from water to an organic liquid is large and negative are undeniable. The former lends credibility to Kauzmann's model, while the latter provides the large negative Gibbs energy change. Although the molecular source of these large negative Gibbs energy changes were not clear, one can always say: "That is an entropy effect" and that is more than enough to silence any objections. I would not go that far as the von Neumann's famous statement that "No one knows what entropy really is" $[17,18]$, however knowing or not knowing what entropy is, the mere invoking of entropy in the "driving force", confers respectability and authority to the person who use it.

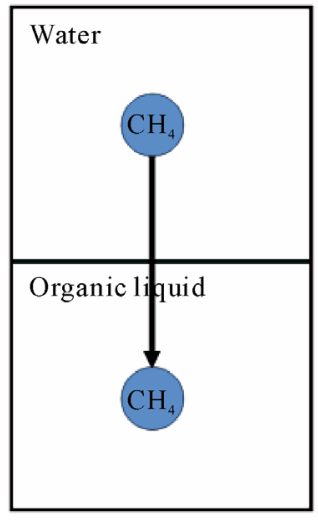

(a)

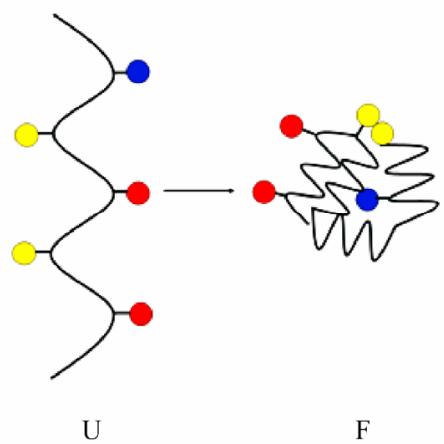

(b)
Figure 1. (a) The process of transferring a methane molecule from water into an organic liquid. (b) The process of transferring a methyl group from water into the interior of the protein. 


\section{The Cracks in the $H \phi O$ Dogma}

As I have said in the previous section, the $H \phi O$ dogma is still alive and thriving. One can find statements about the "dominance" of the $H \phi O$ effect in protein folding even in the most recent reviews and textbooks. Nevertheless, a few whispers of doubts have been heard during the past 20 years.

In 1980, in the preface of my book "Hydrophobic Interactions," I wrote [19]:

"In spite of my researches in this field over almost 10 years, I cannot confirm that there is at present either theoretical or experimental evidence that unequivocally demonstrates the relative importance of the $H \phi O$ interactions over other types of interactions in aqueous solutions."

My doubts were based on lack of evidence in favor of the contention that the $H \phi O$ effect is the most important effect in the "driving force" for protein folding. How can one claim that one factor is more important, or most important when one does not have a full inventory of all the factors involved in protein folding? Remember that Kauzmann's paper was on "some factors in the interpretation of protein denaturation"- - not on all factors involved. No one knew what were all the factors especially those that are solvent-induced. The only factor that could have competed with the $H \phi O$ effect was the $\mathrm{HB}$, but the HB-inventory argument, debilitated the effect of the HBs in aqueous media, and rendered them powerless in explaining the driving force for protein folding.

Kauzmann's model of inference from transferring of molecules from water to organic liquid, and the fact that most $H \phi O$ groups are found in the interior of the protein were so convincing that mere expression of doubts could not have rattled the dominance of the $H \phi O$ dogma. One needs more than doubts. One needs facts! "Lack of evidence" for an idea cannot be used as evidence against that idea.

This was the main motivation for the examination of the entire question of the solvent-induced effects on the protein folding and protein-protein association that I undertook late in the 1980s. The results of this examination were stunning; initially to me, then slowly diffusing into the literature.

First, it was found that the HB-inventory argument was fundamentally faulty [20]. Second, Kauzmann's model, appealing as it was, for over 50 years was found irrelevant to the protein folding process [21-25]. Finally, a logical pitfall: The fact that $H \phi O$ groups are found in the interior of the protein cannot be used as an argument in favor of the role of the $H \phi O$ effect in protein folding.

I shall briefly explain here in qualitative terms each of the abovementioned findings. More can be found in ref- erences [14] and [15]. Recall that the HB-inventory argument was based on the stoichiometric Equation (1). The mere counting of the number of HBs on each side of the equation led to the dismissal of the role of direct hydrogen bonding to the driving force. The first serious challenge to the HB-inventory argument was expressed in 1990 [20]. It was shown that the very writing of the stoichiometric equation in the form (1) is faulty for two reasons:

1) What one loses on the left hand side are not HB energies but solvation Gibbs energies of the $H \phi I$ groups.

2) Whatever the water molecules do when they are released from the solvation sphere of the two $H \phi I$ groups is irrelevant to the driving force. These water molecules "flow" from the solvation sphere into the pool of water at constant chemical potential. Therefore, they cannot contribute anything to the driving force. The stoichiometric reaction must be written instead in the form

$$
\begin{aligned}
& (E-C=O)_{\text {solvated }}+(H N-S)_{\text {solvated }} \\
& \rightarrow(E-C=O \cdots H N-S)_{\text {solvated }}
\end{aligned}
$$

Here, each of the solutes (or the groups) involved in the formation of a HB is solvated by the water molecule. In this form, the HB-inventory argument does not exist. Therefore, the foundations on which the $H \phi O$ has risen have now been demolished. This particular $H \phi I$ effect was estimated to contribute somewhat between -4 and $-6 \mathrm{~kJ} / \mathrm{mol}$ to the driving force of protein folding, for each intramolecular HB formed between two "arms" of the $H \phi I$ groups [20].

Second, the analysis of all the solvent-induced factors revealed that Kauzmann's model does not feature in the "driving force" for the process of protein folding $[22,23]$. Instead of the Gibbs energy of solvation of a $H \phi O$ molecule in water, the conditional solvation Gibbs energy of a $H \phi O$ group features in the "driving force." These Gibbs energies are very different from the Gibbs energies of solvation in water. The main reason is that a $H \phi O$ group attached to the backbone (BB) of the protein is surrounded by water molecules which are perturbed by the BB.

Thus, not only the basis on which the $H \phi O$ model was built upon was demolished, but the Kauzmann's $\mathrm{H} \phi \mathrm{O}$-model itself was now shown to be inadequate $[14,15]$.

Finally, the fact that the $H \phi O$ groups are in the interior of the protein does not necessarily mean that the $\mathrm{H} \phi \mathrm{O}$ effect is the "driving force" for protein folding. "Can anything be more convincing?" Tanford and Reynolds asked rhetorically [19]. Such an inference turned out to be only an illusion [15]. This is exactly the same argument invoked when two different ideal gases mixed spontaneously. The mixing is a fact, but the mixing, in 
itself does not provide the "driving-force" for the mixing process [18].

\section{The Emergence of New $H \phi I$ Effects}

The consequences of the analysis of the solvent-induced effects on protein folding, not only had undermined the foundation on which the $H \phi O$ dogma was erected, and not only demolished the $H \phi O$ dogma itself, but opened the door to a host of new solvent-induced effects that were never considered before. These effects involved $H \phi I$ rather than $H \phi O$ groups. The most important one, and so far the most studied, was the pairwise $H \phi I$ interactions between pairs of $H \phi I$ groups at a distance between $4-5 \AA$. For this particular $H \phi I$ interaction there is overwhelming evidence that it is far stronger than any of the $H \phi O$ effects. The evidence comes from theoretical estimates [14,15], simulations [26,27] and experimental data $[14,15,28]$. There are also some estimates of $H \phi I$ effects involving three and four $H \phi I$ groups. These are more powerful, but probably less frequent [15].

The qualitative explanation of the pairwise $H \phi I$ interaction is quite simple. A $H \phi I$ group is characterized by a few "arms" along which HB may be formed. An amine group on the $\mathrm{BB}$ of the protein has one arm, a carbonyl group has two arms, a hydroxyl group three arms, and a water molecule itself has four arms. When a $H \phi I$ group is in water its arms are solvated by water molecule. The Gibbs energy of solvation per one arm was estimated to be of the order of $-9.4 \mathrm{~kJ} / \mathrm{mol}[14,15]$. Note that this is quite different from a HB energy, as one might have erroneously counted in the HB-inventory argument.

When two such $H \phi I$ groups approach each other to a distance of about $2.8 \AA$ they form a genuine HB. Thus, the Gibbs energy balance is: loss of the solvation Gibbs energy of two arms costs about $20 \mathrm{~kJ} / \mathrm{mol}$, and the formation of a HB provides a HB energy of about -24 $\mathrm{kJ} / \mathrm{mol}$. Therefore, the net change in Gibbs energy for this particular $H \phi I$ effect at a distance of about $2.8 \AA$ is about $-6 \mathrm{~kJ} / \mathrm{mol}[14,15]$.

A more dramatic $H \phi I$ effect was found at a distance of about $4.5 \AA$, the same distance of the second nearest neighbors in ice [25]. When two solvated arms approach each other to this distance, and with the correct orientation, they do not lose their solvation Gibbs energy as in the former $H \phi I$ case. They also do not gain an HB energy. Instead, the mutual salvation Gibbs energy of the pair of $H \phi I$ groups increase by an amount which was estimated to be between -10 and $-12 \mathrm{~kJ} / \mathrm{mol}$.

The reason for such a strong $H \phi I$ interaction is that at this particular configuration the two arms of the two
$H \phi I$ groups can be bridged by a water molecule. It should be stressed however that this effect is not due to a formation of long-lived HB-bridge, as some have misunderstood. Such a "permanent" bridge could provide two HB energies, i.e. about $-48 \mathrm{~kJ} / \mathrm{mol}$. The real effect is a mutual solvation of the two arms of water molecules. This effect involves HB energy, but also involves probability of finding a water molecule that can form a HB-bridge between the two $H \phi I$ groups. The most direct evidence for the existence of such a $H \phi I$ effect is the second peak in the radial distribution function of pure liquid water $[14,15]$. Other experimental evidence comes from the relative solubilities of two isomers of the same molecule, having two $H \phi I$ groups at two different distances $[15,24]$.

Because of the short range of the $\mathrm{HB}$, there exists a steep gradient of the potential of mean force between two $H \phi I$ groups at a distance about $4.5 \AA$. This leads to a strong force between the two $H \phi I$ groups, a force which plays a crucial role in the process of protein folding [15].

One can also think of other $H \phi I$ interactions, one involving one water molecule bridging three $H \phi I$ groups, or two water molecules forming one bridge connecting two $H \phi I$ groups. The former is strong, but rare, the second might be more frequent but very weak. Therefore, it is believed that the pairwise $H \phi I$ interaction at a distance of about $4.5 \AA$ is the more important among the $H \phi I$ effects, hence probably the most important in the process of protein folding as well as in the process of protein-protein association or protein binding to DNA [25].

\section{Dominant Forces and Driving Forces in Protein Folding and in Protein-Protein Association}

In this section, I shall briefly describe how the $H \phi I$ effects may be implemented to understanding the process of protein folding, as well as protein-protein association, protein binding to DNA, self assembly, drug design and many other processes.

To begin with, we must first define the problem of protein folding. In fact, there are several problems associated with protein folding. The most general question asked in this connection is that of the existence of a "code" that translates from the sequence of amino acids into a three dimensional structure. Following the works of Anfinsen [29,30], and others on the spontaneous folding of a denatured protein into the original native structure, it was speculated that the "information" about the folding pathway is already contained in the sequence of amino acids. However, whether or not there exists such a 
"code" that translates from a sequence of amino acids to a 3-D structure of the native protein, what makes the sequence fold into the native structure is the set of forces - not the thermodynamic "driving forces"- that act of each group of the protein. As was shown in reference [15] among all possible forces acting on the groups of the protein, the strongest forces are those between $H \phi I$ groups mediated by the solvent.

The solvent-induced forces are probably the most important factor that governs the process of protein folding. A discussion of the way these forces contribute to the dynamics of protein folding was discussed in references [15] and [25].

Here, I will describe only a few applications of the solvent-induced interactions between $H \phi I$ groups which affect the solvation Gibbs energy of protein. These in turn affects the solubility of proteins, the stability of the native structure of the protein, the stability of dimers or larger aggregates of proteins and molecular recognition.

\subsection{Solvation and Solubility of Proteins}

The high solubility of proteins in water is well known to any biochemist. Yet the molecular reason for the solubility of protein is not less mysterious than the molecular reasons for protein folding and self assembly of biological macromolecules.

The effect of the $H \phi I$ groups on the solubility of protein was long recognized. However, what is less known is that $H \phi I$ interactions are decisive in determining the high solubility of the protein [15,31].

The high solubility of protein is not only the result of the existence of $H \phi I$ groups on the surface of the protein. Furthermore, it is very likely that pairs and higher order correlations between $H \phi I$ groups on the surface of the protein contribute significantly, if not decisively in making the proteins highly soluble.

\subsection{Protein Folding}

In an article entitled "The Problem of How and Why Proteins Adopt Folded Conformations," Creighton [32] discusses the questions of How and Why as if they were one. Clearly, if we knew all the forces acting on all the atoms at each intermediate state of a specific protein, we could answer the question of "Why" and thereby the answer to the question of "How." This answer is pertinent to that specific protein.

An analysis of all the contributions to the solvent-induced effects on the driving forces for the process of protein folding reveals that $H \phi I$ interactions at a distance of about $4.5 \AA$ are probably the strongest.

The first indication that such correlation might be im- portant came from some experimental data published by Haberfield, et al. [28]. These data were used to extract the quantity we shall call the correlation between two $H \phi I$ groups [25].

Soon, more data became available, as well as some simulation of these $H \phi I$ effects, [26,27], and a theoretical estimate of the strength of these effects [25]. All these data, led to the conclusion that correlation between two $H \phi I$ groups (at the correct distance and orientation) are quite significant, and their role in the process of protein folding should be taken more seriously.

The conclusion reached here has some overlaps with the conclusion reached by Rose et al. [33]. Rose et al. proposed an inversion of the "side-chain/backbone paradigm." We advocate the inversion of the $H \phi O / H \phi I$ paradigm. Clearly, since most of the $H \phi I$ are provided by the backbone, it follows that the role of the backbone should be more important than the role of the side chains. In this sense there is an overlap between our proposal and Rose, et al. proposal. However, the two proposals are quite different. This is further discussed in reference [15].

We now turn briefly to discuss the factors involved in the process of protein folding, which are the real physical forces. These forces are the ones that guide the protein in folding along a narrow range of pathways, leading to the native form in a relatively short time $[15,25]$.

We start with Anfinsen's classical work on the renaturation of ribonuclease [29,30]. Anfinsen found that a denatured protein will fold spontaneously when the proper environment for folding are restored (e.g. lowering the temperature or removing a denaturating agent). The folding occurs spontaneously without the need for any additional information beyond that which is contained in the sequence of amino acids.

Thus, the information required for the folding is somehow inscribed in the sequence of the amino acids. Without elaborating on the nature of the information contained in the sequence of amino acids, it is clear that this information is of the type of instructions. These instructions must be read first, then to be executed step by step. The agent that does that job is the "proper environment", and the most important component in this environment is water.

It is believed that water not only "reads" the information contained in the sequence of amino acids but also translates the instruction into executable orders. These "orders" are the forces that are exerted on each of the atoms of the protein that causes the motion of the entire protein towards the end product. Because of the statistical character of these forces the motion of the protein is not along a unique deterministic route, but along a narrow range of routes or pathways. 
Most reviews on protein folding focus on the thermodynamic "driving forces" rather than the forces themselves [34]. Recently, an analysis of the types of forces acting on the protein, and more specifically on the solvent-induced forces in protein folding, was undertaken $[15,25]$.

\subsection{Self Assembly and Molecular Recognition}

Binding, association and self assembly processes abound in biological systems. These processes range from binding small ligands such as drugs to protein or to DNA, association between proteins, as in hemoglobin and self-assembly of a large number of subunits to form macromolecules such as Tobacco mosaic virus.

There are essentially two puzzles associated with these processes. The first is similar to the question of the stability of the folded protein. In the folding process, the large number of conformational states of the unfolded form tends to favor the denatured protein. To understand the "driving force" for folding we need to find out the factors that stabilize the native structure of the protein. Similarly, in any association process there are far more configurations to the separated units than to the bound aggregates. Again, to understand the "driving force" for the binding process, we must find out which factors are responsible for the stabilization of the dimer or the oligomer, relative to the separate monomers.

The second puzzle is similar to the preferential pathways of protein folding. It is concerned with the specificity of the binding mode. There are many ways two globular proteins can bind, yet only one specific binding mode is stable. Specificity of binding is essentially the same as molecular recognition. These phenomena are relevant for diverse biochemical systems ranging from binding drugs to protein (hence also to drug design), binding of protein to DNA (controlling genetic expression), and the way the immune system works [35].

We have found that the $H \phi I$ interactions could be decisive in determining both the stability and the specificity of the mode of association between two proteins.

The finding that $H \phi I$ interactions can change the preferential binding site has far reaching consequences to the problem of drug design, either for designing new drugs or for modifying existing drugs to improve their efficacy. Some specific examples were discussed recently [36]. We shall not present these highly technical examples here. The interested reader should consult the article by Wang and Ben-Naim [36].

\section{Conclusions}

The paradigm change from the $H \phi O$ to $H \phi I$ effects has brought us as close as one can hope for, to the solution of the problem of protein folding and self assembly of proteins.

\section{References}

[1] L. Pauling, "The Nature of Chemical Bond," 2nd Edition, Cornell University Press, Ithaca, 1948.

[2] L. Pauling, "The Nature of Chemical Bond," 3rd Edition, Cornell University Press, Ithaca, 1960.

[3] M. L. Anson and A. E. Mirsky, "The Equilibria between Native and Denatured Hemoglobin in Salicylate Solutions and the Theoretical Consequences of the Equilibrium between Native and Denatured Protein," Journal General Physiology, Vol. 17, No. 3, 1934, pp. 393-408. doi:10.1085/igp.17.3.393

[4] A. E. Mirsky and L. Pauling, "On the Structure of Native, Denatured and Coagulated Proteins," Proceedings of the National Academy Science of the USA, Vol. 22, No. 7, 1936, pp. 439-447. doi:10.1073/pnas.22.7.439

[5] L. Pauling and R. B. Corey, "Atomic Coordinates and Structure Factors for Two Helical Configurations of Polypeptide Chains," Proceedings of the National Academy Science of the USA, Vol. 37, No. 5, 1951, pp. 235-248. doi:10.1073/pnas.37.5.235

[6] L. Pauling and R. B. Corey, "The Pleated Sheet, A New Layer Configuration of Polypeptide Chains," Proceedings of the National Academy Science, USA, Vol. 37, No. 5, 1951, pp. 251-256. doi:10.1073/pnas.37.5.251

[7] L. Pauling and R. B. Corey, "The Structure of Fibrous Proteins of the Collagen-Gelatin Group," Proceedings of the National Academy Science of the USA, Vol. 37, No. 5, 1951, pp. 272-281. doi:10.1073/pnas.37.5.272

[8] L. Pauling and R. B. Corey, "Configurations of Peptide Chains with Favored Orientations around Single Bonds: Two New Pleated Sheets," Proceedings of the National Academy Science of the USA, Vol. 37, No. 5, 1951, pp. 729-740.

[9] C. Tanford and J. Reynold, "Nature's Robots, A History of Proteins," Oxford University Press, Oxford, 2003.

[10] J. A. Schellmann, "The Thermodynamics of Urea Solutions and the Heat of Formation of the Peptide Hydrogen Bond," Comptes Rendus des Travaux du Laboratoire Carlsberg, Serie Chimique, Vol. 29, No. 14-15, 1955, pp. 223-230.

[11] J. A. Schellmann, "The Stability of Hydrogen-Bonded Peptide Structures in Aqueous Solution," Comptes Rendus des Travaux du Laboratoire Carlsberg, Serie Chimique, Vol. 29, No. 14-15, 1955, pp. 230-259.

[12] W. Kauzmann, "Some Factors in the Interpretation of Protein Denaturation," Advances in Protein Chemistry, Vol. 14, 1959, pp. 1-63.

doi:10.1016/S0065-3233(08)60608-7

[13] A. Fersht, "Structure and Mechanism in Protein Science," W. H. Freeman and Company, New York, 1999.

[14] A. Ben-Naim, "Molecular Theory of Water and Aqueous 
Solutions, Part I: Understanding Water," World Scientific, Singapore, 2009. doi:10.1142/9789812837615

[15] A. Ben-Maim, "Molecular Theory of Water and Aqueous Solutions, Part II: Hydrophilic Effects in Protein Folding, Self-Assembly and Molecular Recognition," World Scientific, Singapore, 2011.

[16] A. Ben-Naim, "Entropy Demystified. The Second Law Reduced to Plain Common Sense," World Scientific, Singapore, 2007. doi:10.1142/9789812770691

[17] M. Tribus and McIrvine, "Energy and Information," Scientific American, Vol. 225, No. 3, 1971, pp. 179-188. doi:10.1038/scientificamerican0971-179

[18] A. Ben-Naim, "A Farewell to Entropy. Statistical Mechanics Based on Information," World Scientific, Singapore, 2008.

[19] A. Ben-Naim, "Hydrophobic Interactions," Plenum Press, New York, 1980.

[20] A. Ben-Naim, "On the Role of Hydrogen-Bonds in Protein Folding and Protein Association," Journal of Physical Chemistry, Vol. 95, No. 3, 1990, pp. 1444-1473.

[21] A. Ben-Naim, "Solvent-Induced Interactions: Hydrophobic and Hydrophilic Phenomena," Journal of Chemical Physics, Vol. 90, No. 12, 1989, pp. 7412-7525. doi:10.1063/1.456221

[22] A. Ben-Naim, "Solvent Effects on Protein Association and Protein Folding," Biopolymers, Vol. 29, No. 3, 1990, pp. 567-596. doi:10.1002/bip.360290312

[23] A. Ben-Naim, "Solvent Induced Forces in Protein Folding," Journal of Chemical Physics, Vol. 94, No. 17, 1990, pp. 6893-6895. doi:10.1021/j100380a065

[24] A. Ben-Naim, "Strong Forces between Hydrophilic Macromolecules; Implications in Biological Systems," Journal of Chemical Physics, Vol. 93, No. 11, 1991, pp. 8196-8210. doi:10.1063/1.459298

[25] A. Ben-Naim, "Statistical Thermodynamics for Chemists and Biochemists," Plenum Press, New York, 1992.

[26] M. Mezei and A. Ben-Naim, "Calculation of the Solvent Contribution to the Potential of Mean Force between
Water Molecules in Fixed Relative Orientation in Liquid Water," Journal of Chemical Physics, Vol. 92, No. 2, 1990, pp. 1359-1361. doi:10.1063/1.458146

[27] S. R. Durell, B. R. Brooks and A. Ben-Naim, "Solvent-Induced Forces between Two Hydrophilic Groups," Journal of Physical Chemistry, Vol. 98, No. 8, 1994, pp. 2198-2202. doi:10.1021/j100059a038

[28] P. Haberfield, J. Kivuls, M. Haddad and T. Rizzo, "Enthalpies, Free Energies and Entropies of Transfer of Phenols from Nonpolar Solvents to Water," Journal of Physical Chemistry, Vol. 88, No. 9, 1984, pp. 1913-1916. doi:10.1021/j150653a049

[29] C. B. Anfinsen, "Principles that Govern the Folding of Protein Chains," Science, New Series, Vol. 181, No. 4096, 1973, pp. 223-230.

[30] E. Haber and C. B. Anfinsen, "Studies on the Reduction on Reformation of Protein Disulfide Bonds," Journal of Biological Chemistry, Vol. 236, 1961, pp. 1361-1363.

[31] H. Wang and A. Ben-Naim, "Solvation and Solubility of Globular Proteins," Journal of Physical Chemistry B, Vol. 101, No. 6, 1997, pp. 1077-1086. doi:10.1021/jp961591b

[32] T. E. Creighton, "The Problem of How and Why Proteins Adopt Folded Conformations," Journal of Physical Chemistry, Vol. 89, No. 12, 1985, pp. 2452-2459. doi:10.1021/j100258a006

[33] G. D. Rose, P. J. Fleming, J. R. Banavar and A. Maritan, "A Backbone Based Theory of Protein Folding," Proceedings of the National Academy of Science, Vol. 103, No. 45, 2006, pp. 16623-16633. doi: $10.1073 /$ pnas.0606843103

[34] K. A. Dill, "Dominant Forces in Protein Folding," Biochemistry, Vol. 29, No. 31, 1990, pp. 7133-7155. doi:10.1021/bi00483a001

[35] A. Ben-Naim, "Cooperativity and Regulation in Biochemical Processes," Kluwer Academic/Plenum Publishers, New York, 2001.

[36] H. Wang and A. Ben-Naim, "A Possible Involvement of Solvent Induced Interactions in Drug Design," Journal of Medicinal Chemistry, Vol. 39, No. 7, 1996, pp 1531-1539. doi:10.1021/jm950430d 